\title{
THE GROTHENDIECK RING OF VARIETIES IS NOT A DOMAIN
}

\author{
BJORN POONEN
}

\begin{abstract}
If $k$ is a field, the ring $K_{0}\left(\mathcal{V}_{k}\right)$ is defined as the free abelian group generated by the isomorphism classes of geometrically reduced $k$-varieties modulo the set of relations of the form $[X-Y]=[X]-[Y]$ whenever $Y$ is a closed subvariety of $X$. The multiplication is defined using the product operation on varieties. We prove that if the characteristic of $k$ is zero, then $K_{0}\left(\mathcal{V}_{k}\right)$ is not a domain.
\end{abstract}

\section{The Grothendieck ring of varieties}

Let $k$ be a field. By a $k$-variety we mean a geometrically reduced, separated scheme of finite type over $k$. Let $\mathcal{V}_{k}$ denote the category of $k$-varieties. Let $K_{0}\left(\mathcal{V}_{k}\right)$ denote the free abelian group generated by the isomorphism classes of $k$-varieties, modulo all relations of the form $[X-Y]=[X]-[Y]$ where $Y$ is a closed $k$-subvariety of a $k$-variety $X$. Here, and from now on, $[X]$ denotes the class of $X$ in $K_{0}\left(\mathcal{V}_{k}\right)$. The operation $[X] \cdot[Y]:=\left[X \times_{k} Y\right]$ is well-defined, and makes $K_{0}\left(\mathcal{V}_{k}\right)$ a commutative ring with 1 . It is known as the Grothendieck ring of $k$-varieties. A completed localization of $K_{0}\left(\mathcal{V}_{k}\right)$ is needed for the theory of motivic integration, which has many applications: see [Loo00] for a survey.

Our main result is the following.

Theorem 1. Suppose that $k$ is a field of characteristic zero. Then $K_{0}\left(\mathcal{V}_{k}\right)$ is not a domain.

Remark. We conjecture that the result holds also for fields $k$ of characteristic $p$. But we use a result whose proof relies on resolution of singularities and weak factorization of birational maps, which are known only in characteristic zero.

\section{Abelian varieties of $\mathrm{GL}_{2}$-type}

If $A$ is an abelian variety over a field $k_{0}$, and $k$ is a field extension of $k_{0}$, then $\operatorname{End}_{k}(A)$ denotes the endomorphism ring of the base extension $A_{k}:=A \times_{k_{0}} k$, that is, the ring of endomorphisms defined over $k$.

Received June 8, 2002.

2000 Mathematics Subject Classification. Primary 14A10; Secondary 14G35.

Key words and phrases. Grothendieck ring of varieties, modular abelian variety, stable birational equivalence, Albanese variety.

This research was supported by NSF grant DMS-9801104, and a Packard Fellowship. 
Lemma 2. Let $k$ be a field of characteristic zero, and let $\bar{k}$ denote an algebraic closure. There exists an abelian variety $A$ over $k$ such that $\operatorname{End}_{k}(A)=$ $\operatorname{End}_{\bar{k}}(A) \simeq \mathcal{O}$, where $\mathcal{O}$ is the ring of integers of a number field of class number 2 .

Let us precede the proof of Lemma 2 with a few paragraphs of motivation. Our strategy will be to find a single abelian variety $A$ over $\mathbb{Q}$ such that the base extension $A_{k}$ works over $k$.

Let $A$ be a simple abelian variety over $\mathbb{Q}$. Let $E=\operatorname{End}_{\mathbb{Q}}(A) \otimes \mathbb{Q}$. Since $A$ is simple, $E$ is a division algebra. The Lie algebra Lie $A$ is a nonzero left $E$-vector space, so $[E: \mathbb{Q}] \leq \operatorname{dim}_{\mathbb{Q}} \operatorname{Lie} A=\operatorname{dim} A$. If equality holds and $E$ is commutative (hence a number field), then $A$ is said to be of $\mathrm{GL}_{2}$-type. (The terminology is due to the following: If $A$ is of $\mathrm{GL}_{2}$-type, then the action of the Galois group $\operatorname{Gal}(\overline{\mathbb{Q}} / \mathbb{Q})$ on a Tate module $V_{\ell} A$ can be viewed as a representation $\rho_{\ell}: \operatorname{Gal}(\overline{\mathbb{Q}} / \mathbb{Q}) \rightarrow \mathrm{GL}_{2}\left(E \otimes \mathbb{Q}_{\ell}\right)$. $)$

Because $\mathbb{Q}$ has class number 1 , we must take $[E: \mathbb{Q}] \geq 2$ to find an $A$ over $\mathbb{Q}$ as in Lemma 2. The inequality $\operatorname{dim} A \geq[E: \mathbb{Q}]$ then forces $\operatorname{dim} A \geq 2$. Moreover, if we want $\operatorname{dim} A=2$, then $A$ must be of $\mathrm{GL}_{2}$-type.

Abelian varieties of $\mathrm{GL}_{2}$-type are closely connected to modular forms. For each $N \geq 1$, let $\Gamma_{1}(N)$ denote the classical modular group, let $X_{1}(N)$ denote the corresponding modular curve over $\mathbb{Q}$, and let $J_{1}(N)$ be the Jacobian of $X_{1}(N)$. G. Shimura, in Theorem 1 of [Shi73], attached to each weight- 2 newform $f$ on $\Gamma_{1}(N)$ an abelian variety quotient $A_{f}$ of $J_{1}(N)$. (Previously, in Theorem 7.14 of [Shi71], he had attached to $f$ an abelian subvariety of $J_{1}(N)$.) Let $E_{f}$ be the number field generated over $\mathbb{Q}$ by the Fourier coefficients of $f$. Theorem 1 of [Shi73] shows also that $\operatorname{dim} A_{f}=\left[E_{f}: \mathbb{Q}\right]$, and that there is an injective $\mathbb{Q}$-algebra homomorphism $\theta: E_{f} \hookrightarrow E:=\operatorname{End}_{\mathbb{Q}}\left(A_{f}\right) \otimes \mathbb{Q}$ mapping each Fourier coefficient to the endomorphism of $A_{f}$ induced by the associated Hecke correspondence on $X_{1}(N)$. Corollary 4.2 of [Rib80] proves that $\theta$ is an isomorphism. It follows that $A_{f}$ is of $\mathrm{GL}_{2}$-type.

Conversely, it is conjectured that each simple abelian variety over $\mathbb{Q}$ of $\mathrm{GL}_{2}$ type is $\mathbb{Q}$-isogenous to some $A_{f}$. See [Rib92] for more details. The $\operatorname{dim} A=1$ case of this conjecture is the statement that elliptic curves over $\mathbb{Q}$ are modular, which is known [BCDT01].

Therefore we are led to consider $A_{f}$ of dimension 2, where $f$ is a newform as above.

Proof of Lemma 2. Tables [Ste] show that there exists a weight-2 newform $f=$ $\sum_{n=1}^{\infty} a_{n} q^{n}$ on $\Gamma_{0}(590)$ (hence also on $\left.\Gamma_{1}(590)\right)$ such that $E_{f}=\mathbb{Q}(\sqrt{10})$ and $a_{3}=\sqrt{10}$. Let $A=A_{f}$ be the corresponding abelian variety over $\mathbb{Q}$. Then $\operatorname{dim} A=\left[E_{f}: \mathbb{Q}\right]=2 . \operatorname{But}_{\operatorname{End}_{\mathbb{Q}}}(A)$ is an order of $E=E_{f}$ containing $a_{3}=\sqrt{10}$, so $\operatorname{End}_{\mathbb{Q}}(A)$ is the maximal order $\mathbb{Z}[\sqrt{10}]$ of $E$. Since 590 is squarefree, $A$ is semistable over $\mathbb{Q}$ by Theorem 6.9 of [DR73], and then Corollary 1.4(a) of [Rib75] shows that all endomorphisms of $A$ over any field extension $k$ of $\mathbb{Q}$ are defined over $\mathbb{Q}$. Finally, the class number of $\mathbb{Z}[\sqrt{10}]$ is 2 . 


\section{Remarks.}

1. After one knows that $\operatorname{End}_{\mathbb{Q}}(A)=\mathbb{Z}[\sqrt{10}]$, another way to prove $\operatorname{End}_{\overline{\mathbb{Q}}}(A)=$ $\mathbb{Z}[\sqrt{10}]$ is to use the fact that $\operatorname{End}_{\overline{\mathbb{Q}}}(A)$ injects into the endomorphism ring of the reduction $A_{p}$ over $\overline{\mathbb{F}}_{p}$ for any prime $p$ not dividing 590 . The latter endomorphism rings can be computed using Eichler-Shimura theory and Honda-Tate theory. Combining the information from a few primes $p$ yields the result.

2. The smallest $N$ for which there exists a newform $f$ on $\Gamma_{0}(N)$ with $E_{f}$ of class number 2 is 276 . The advantage of 590 is that it is squarefree. (In fact, our original proof applied the technique in the previous remark at level 276.)

3. The case $k=\mathbb{C}$ of Lemma 2 has an easy proof: let $A$ be an elliptic curve over $\mathbb{C}$ with complex multiplication by $\mathbb{Z}[\sqrt{-5}]$.

\section{Abelian varieties and projective modules}

Let $A$ be an abelian variety over a field $k$, and let $\mathcal{O}=\operatorname{End}_{k}(A)$. Given a finite-rank projective right $\mathcal{O}$-module $M$, we define an abelian variety $M \otimes_{\mathcal{O}} A$ as follows: choose a finite presentation $\mathcal{O}^{m} \rightarrow \mathcal{O}^{n} \rightarrow M \rightarrow 0$, and let $M \otimes_{\mathcal{O}} A$ be the cokernel of the homomorphism $A^{m} \rightarrow A^{n}$ defined by the matrix that gives $\mathcal{O}^{m} \rightarrow \mathcal{O}^{n}$. It is straightforward to check that this is independent of the presentation, and that $M \mapsto\left(M \otimes_{\mathcal{O}} A\right)$ defines a fully faithful functor $T$ from the category of finite-rank projective right $\mathcal{O}$-modules to the category of abelian varieties over $k$. (Essentially the same construction is discussed in the appendix by J.-P. Serre in [Lau01].)

Lemma 3. Let $k$ be a field of characteristic zero. There exist abelian varieties $A$ and $B$ over $k$ such that $A \times A \simeq B \times B$ but $A_{\bar{k}} \neq B_{\bar{k}}$.

Proof. Let $A$ and $\mathcal{O}$ be as in Lemma 2. Let $I$ be a nonprincipal ideal of $\mathcal{O}$. Since $\mathcal{O}$ is a Dedekind domain, the isomorphism type of a direct sum of fractional ideals $I_{1} \oplus \ldots \oplus I_{n}$ is determined exactly by the nonnegative integer $n$ and the product of the classes of the $I_{i}$ in the class group $\operatorname{Pic}(\mathcal{O})$. Since $\operatorname{Pic}(\mathcal{O}) \simeq \mathbb{Z} / 2$, we have $\mathcal{O} \oplus \mathcal{O} \simeq I \oplus I$ as $\mathcal{O}$-modules. Applying the functor $T$ yields $A \times A \simeq B \times B$, where $B:=I \otimes_{\mathcal{O}} A$. Since $\operatorname{End}_{\bar{k}}(A)$ also equals $\mathcal{O}$, we have $B_{\bar{k}}=I \otimes_{\mathcal{O}} A_{\bar{k}}$. Since $T$ for $\bar{k}$ is fully faithful, $A_{\bar{k}} \nsucceq B_{\bar{k}}$.

\section{Stable birational classes and Albanese varieties}

For any extension of fields $k \subseteq k^{\prime}$, there is a ring homomorphism $K_{0}\left(\mathcal{V}_{k}\right) \rightarrow$ $K_{0}\left(\mathcal{V}_{k^{\prime}}\right)$ mapping $[X]$ to $\left[X_{k^{\prime}}\right]$.

Let $k$ be a field of characteristic zero. Smooth, projective, geometrically integral $k$-varieties $X$ and $Y$ are called stably birational if $X \times \mathbb{P}^{m}$ is birational to $Y \times \mathbb{P}^{n}$ for some integers $m, n \geq 0$. The set $\mathrm{SB}_{k}$ of equivalence classes of this relation is a monoid under product of varieties over $k$. Let $\mathbb{Z}\left[\mathrm{SB}_{k}\right]$ denote the corresponding monoid ring. 
When $k=\mathbb{C}$, there is a unique ring homomorphism $K_{0}\left(\mathcal{V}_{k}\right) \rightarrow \mathbb{Z}\left[\mathrm{SB}_{k}\right]$ mapping the class of any smooth projective integral variety to its stable birational class [LL01]. (In fact, this homomorphism is surjective, and its kernel is the ideal generated by $\mathbb{L}:=\left[\mathbb{A}^{1}\right]$.) The proof in [LL01] requires resolution of singularities and weak factorization of birational maps [AKMW00, Theorem 0.1.1], [Wło01, Conjecture 0.0.1]. The same proof works over any algebraically closed field of characteristic zero.

The set $\mathrm{AV}_{k}$ of isomorphism classes of abelian varieties over $k$ is a monoid. The Albanese functor mapping a smooth, projective, geometrically integral variety to its Albanese variety induces a homomorphism of monoids $\mathrm{SB}_{k} \rightarrow \mathrm{AV}_{k}$, since the Albanese variety is a birational invariant, since formation of the Albanese variety commutes with products, and since the Albanese variety of $\mathbb{P}^{n}$ is trivial. Therefore we obtain a ring homomorphism $\mathbb{Z}\left[\mathrm{SB}_{k}\right] \rightarrow \mathbb{Z}\left[\mathrm{AV}_{k}\right]$.

\section{Zerodivisors}

Proof of Theorem 1. Let $A$ and $B$ be as in Lemma 3. Then $([A]+[B])([A]-[B])$ $=0$ in $K_{0}\left(\mathcal{V}_{k}\right)$. On the other hand, $[A]+[B]$ and $[A]-[B]$ are nonzero, because their images under the composition

$$
K_{0}\left(\mathcal{V}_{k}\right) \rightarrow K_{0}\left(\mathcal{V}_{\bar{k}}\right) \rightarrow \mathbb{Z}\left[\mathrm{SB}_{\bar{k}}\right] \rightarrow \mathbb{Z}\left[\mathrm{AV}_{\bar{k}}\right]
$$

are nonzero. (The Albanese variety of an abelian variety is itself.)

\section{Acknowledgements}

I thank Ken Ribet for several comments regarding Section 2, and in particular for suggesting a less computational proof of Lemma 2. I thank also Eduard Looijenga and Arthur Ogus for discussions. The tables [Ste] were developed using MAGMA, C++, LiDIA, and GP-PARI. The package GP-PARI was used also to search the data from [Ste] for a newform $f$ suitable for the proof of Lemma 2.

\section{References}

[AKMW00] Dan Abramovich, Kalle Karu, Kenji Matsuki, and Jarosław Włodarczyk, Torification and factorization of birational maps, J. Amer. Math. Soc. 15 (2002), 531-572.

[BCDT01] Christophe Breuil, Brian Conrad, Fred Diamond, and Richard Taylor, On the modularity of elliptic curves over $\mathbf{Q}$ : wild 3-adic exercises, J. Amer. Math. Soc. 14 (2001), 843-939 (electronic).

[DR73] P. Deligne and M. Rapoport, Les schémas de modules de courbes elliptiques, Modular functions of one variable, II. Proc. Internat. Summer School, Univ. Antwerp, Antwerp, 1972, pp. 143-316, Lecture Notes in Math., Vol. 349, Springer, Berlin, 1973.

[Lau01] Kristin Lauter, The maximum or minimum number of rational points on curves of genus three over finite fields, with an appendix by Jean-Pierre Serre, preprint, 2001; math. AG/0104086

[LL01] Michael Larsen and Valery A. Lunts, Motivic measures and stable birational geometry, preprint, 2001; math.AG/0110255 
[Loo00] Eduard Looijenga, Motivic measures, Séminaire Bourbaki, Vol. 1999/2000. Astérisque No. 276, (2002), 267-297.

[Rib75] Kenneth A. Ribet, Endomorphisms of semi-stable abelian varieties over number fields, Ann. Math. (2) 101 (1975), 555-562.

[Rib80] , Twists of modular forms and endomorphisms of abelian varieties, Math. Ann. 253 (1980), 43-62.

[Rib92] _ Abelian varieties over $\mathbf{Q}$ and modular forms, Algebra and topology 1992 (Taejŏn), 53-79, Korea Adv. Inst. Sci. Tech., Taejŏn, 1992.

[Shi71] Goro Shimura, Introduction to the arithmetic theory of automorphic functions, Kanô Memorial Lectures, No.1. Publications of the Mathematical Society of Japan, No. 11. Iwanami Shoten, Publishers, Tokyo; Princeton University Press, Princeton, NJ, 1971.

[Shi73] On the factors of the jacobian variety of a modular function field, J. Math. Soc. Japan 25 (1973), 523-544.

[Ste] William Stein, The Modular Forms Database, http://modular.fas.harvard.edu/ Tables

[Wło01] Jarosław Włodarczyk, Toroidal varieties and the weak factorization theorem, preprint, 2001; math.AG/9904076

Department of Mathematics, University of California, Berkeley, CA 94720-3840, U.S.A.

E-mail address: poonen@math.berkeley.edu 